TITLE:

\title{
Shift and broadening in attenuated total reflection spectra of the hyperfine-structure-resolved D-2 line of dense rubidium vapor
}

\section{AUTHOR(S):}

Kondo, R; Tojo, S; Fujimoto, T; Hasuo, M

\section{CITATION:}

Kondo, R ... [et al]. Shift and broadening in attenuated total reflection spectra of the hyperfine-structure-resolved D-2 line of dense rubidium vapor. PHYSICAL REVIEW A 2006, 73(6): 062504.

ISSUE DATE:

2006-06

URL:

http://hdl.handle.net/2433/39834

RIGHT:

Copyright 2006 American Physical Society 


\title{
Shift and broadening in attenuated total reflection spectra of the hyperfine-structure-resolved $\mathrm{D}_{2}$ line of dense rubidium vapor
}

\author{
Rikio Kondo, Satoshi Tojo, ${ }^{*}$ Takashi Fujimoto, and Masahiro Hasuo \\ Department of Engineering Physics and Mechanics, Graduate School of Engineering, Kyoto University, Kyoto 606-8501, Japan
}

(Received 14 February 2005; published 8 June 2006)

\begin{abstract}
We have observed attenuated total reflection at a glass/rubidium vapor interface in high precision. The atom density was from $3.8 \times 10^{20}$ to $3.7 \times 10^{21} \mathrm{~m}^{-3}$ and the angle of incidence was from 48.4 to $64.8^{\circ}$. Redshift and broadening of the reflection spectra were observed. The redshift depended on atom density, angle of incidence, light polarization and hyperfine structure. The observed broadening was attributed to the self-broadening of the resonance line. Calculation based on the Fresnel formula with a local index for the susceptibility of the vapor, which included the Lorentz local field correction and the modification of the wave vector of the evanescent light by the optically dense atomic vapor, was found to be in reasonable agreement with the experimental results.
\end{abstract}

DOI: 10.1103/PhysRevA.73.062504

PACS number(s): 32.30.Jc, 32.70.Cs, 42.62.Fi

\section{INTRODUCTION}

Reflection spectroscopy with a dielectric/vapor interface is known to be useful in investigations of atoms near a surface. In the case of normal incidence of the light to the interface, a sub-Doppler structure has been observed. This is due to the transient behavior of atoms leaving the surface after a collision on the surface and is called selective reflection [1,2]. Another reflection spectroscopy is based on the attenuated total reflection (ATR), in which the evanescent light is thought to give rise to absorption [3-10]. The evanescent light penetrates into the vapor with a thickness of less than the light wavelength.

In a high atom density regime or an optically dense regime, shift and broadening of alkali atom D lines have been measured by selective reflection spectroscopy $[11,12]$. In order to interpret the shift and broadening, several theoretical investigations, which include the Lorentz local field and selfconsistent treatment of the interaction between the vapor and the light field, have been carried out [13-15]. Since the light field, which interacts with atoms in the vapor in selective reflection spectroscopy, is in the propagating mode, the effect of the atom vapor on the light field, especially on the propagation characteristics, is an area of research of current interest.

The light field, which interacts with atoms in total reflection spectroscopy, is in the evanescent mode or the localized mode at the interface. Usually, characteristics of the evanescent light field are assumed to be determined by the refractive indices of the interface and the angle of incidence of light [3-9]. There are few reports about the effects of an optically dense vapor on ATR spectra.

Recently we observed a redshift in the ATR spectra of the rubidium $\mathrm{D}_{2}$ line and found that the calculated spectra with the Lorentz local field correction reproduced the redshift only partly [8]. In this article, we present our systematic measurement of the ATR spectra of the rubidium $\mathrm{D}_{2}$ line up

*Present address: Department of Physics, Gakushuin University, Tokyo 171-8588, Japan. to the atom density one order of magnitude larger than that in Ref. [8], where the attenuation is as large as $80 \%$ at the minimum of the ATR spectra. We also present calculated spectra with the Fresnel formula with a local index of the vapor modified by the Lorentz local field correction and the change of the wave vector of the evanescent light by the vapor, the effect of which may be salient in an optically dense regime.

\section{EXPERIMENTAL SETUP}

Figure 1 shows the schematic diagram of our experimental setup. The light source is an external-cavity controlled diode laser (EOSI, 2010). We scan the laser light frequency over the rubidium $\mathrm{D}_{2}$ line $(\lambda=780 \mathrm{~nm})$ by sweeping the voltage applied to the piezoelectric element, which controls the external cavity. The applied voltage is swept by a personal computer with a digital-to-analog converter. After passing through an optical isolator, a part of the light beam is incident on a Fabry-Pérot frequency marker with a free spectral range of $1.870 \pm 0.004 \mathrm{GHz}$; we calibrated this value against the separation $(1.124 \mathrm{GHz})$ between the ${ }^{85} \mathrm{Rb}\left(F=3-F^{\prime}=4\right)$ peak and the ${ }^{87} \mathrm{Rb}\left(F=2-F^{\prime}=3\right)$ peak in the saturated absorption spectrum using a 5-cm-long reference rubidium cell

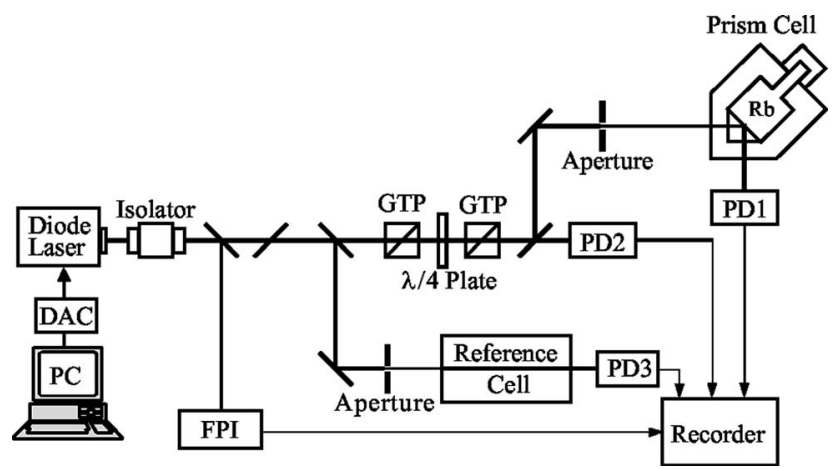

FIG. 1. The experimental setup. DAC: digital-to-analog converter, FPI: Fabry-Pérot interferometer as a frequency marker, GTP: Glan-Thompson prism, PD: photo detector. 

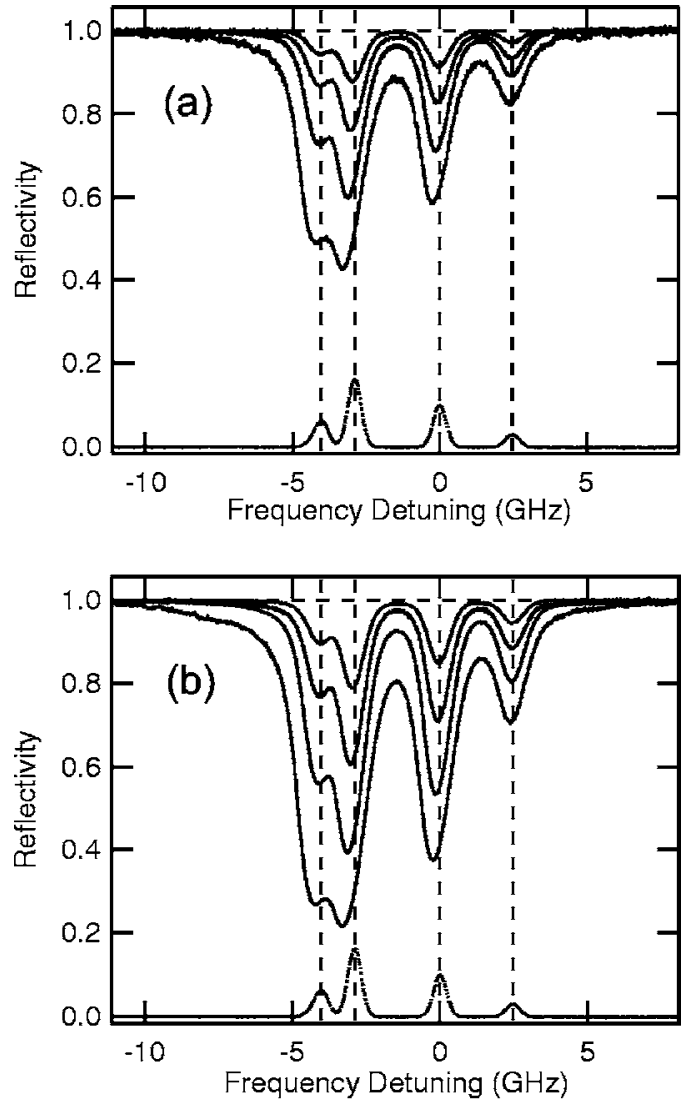

(c)
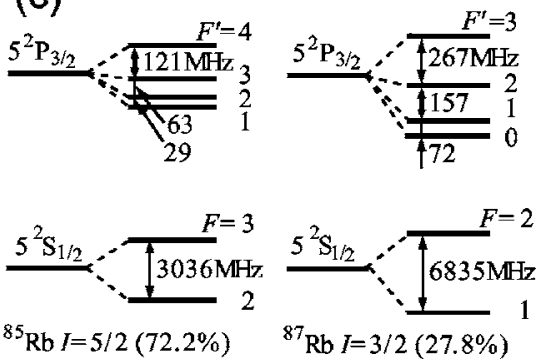

FIG. 2. ATR spectra at $\theta_{1}=48.4^{\circ}$ for (a) $s$ and (b) $p$ polarizations at the coldest temperature 453, 473, 493 and $513 \mathrm{~K}$ (the solid curves from top to bottom). The dotted curve is the absorption spectrum of the reference cell at room temperature. The vertical dashed lines show the peak positions of the absorption spectrum. (c) Energy level diagram of the $\mathrm{Rb} \mathrm{D}_{2}$ line with hyperfine structure splitting.

at room temperature. The main part of the laser beam goes through a Glan-Thompson prism and a quarter wave plate. The light is thus circularly polarized. By another GlanThompson prism, the laser light is polarized horizontally ( $p$ polarization) or vertically ( $s$ polarization) with almost equal intensity. It goes through an aperture (1 mm diameter) and is incident on a prism of Pyrex glass whose refractive index is 1.472 at $780 \mathrm{~nm}$, giving the critical angle for the total internal reflection, $\theta_{c}$, of $42.79^{\circ}$ at the glass/vacuum interface. This prism serves as one side of the cell, which contains rubidium vapor. This cell, hereafter referred to as the prism cell, is enclosed by a cover cell whose entrance and exit windows are wedged to eliminate the interference between the reflected light by the outer and inner surfaces. The intensity of the laser light beam incident on the prism is $16 \mu \mathrm{W} / \mathrm{mm}^{2}$.

The angles of incidence for reflection at the glass/vapor interface, $\theta_{1}$, range from 48.4 to $64.8^{\circ}$. The main body of the prism cell and the leg ( $20 \mathrm{~mm}$ long) on the opposite side to the prism are heated independently, so that the temperature of the main body is uniform and the leg serves as the coldest point, which determines the vapor pressure of rubidium. The temperature of the coldest point is set at 453, 473, 493 or $513 \mathrm{~K}$ and the temperature of the main body is $10 \mathrm{~K}$ higher than the coldest point temperature. The accuracy of the measured temperatures is within $0.5 \mathrm{~K}$. The totally reflected light is detected by a photodiode (Hamamatsu, S1336-5KB). A part of the incident light is detected by another photodiode for the purpose of normalization of the reflected light intensity. Outputs of the photodiodes are registered by a digital recorder. The frequency of the laser light is calibrated against the transmission spectrum of the reference rubidium cell and the output of the Fabry-Pérot frequency marker, both registered simultaneously. We confirm that the experiment is free from any effects due to the magnetic field caused by the heater current and any saturation effects caused by the finite intensity of the excitation light.

\section{RESULTS AND DISCUSSION}

The solid curves in Fig. 2(a) and 2(b) show the observed ATR spectra at $\theta_{1}=48.4^{\circ}$ for $s$ and $p$ polarizations, respectively. The dotted curve is the absorption spectrum obtained from the transmission spectrum of the reference cell. The vertical dashed lines show the positions of the absorption peaks of the reference cell. The observed four absorption peaks correspond to the hyperfine structure (HFS) splittings of the ground state level $5{ }^{2} S_{1 / 2}$ of the two major rubidium isotopes as shown in Fig. 2(c). The HFS splittings of the excited level $5^{2} P_{3 / 2}$ are too small to be resolved in our spectrum owing mainly to the Doppler and transit-time broadening $[3,4]$. With an increase in the coldest point temperature, or the atom density, the redshift of each absorption peak in the ATR spectra increases for both polarizations. The magnitude of the shift depends on the HFS components. The observed increase of the absorption tail at higher temperatures suggests the presence of a Lorentzian type broadening, which depends on atom density.

Within the framework of the index theory, the Fresnel formula gives a reflection profile as [16]

$$
\mathcal{R}_{s, p}=\left|\frac{n_{1} \cos \theta_{1}-n_{s, p}^{2} n_{2} \cos \theta_{2}}{n_{1} \cos \theta_{1}+n_{s, p}^{2} n_{2} \cos \theta_{2}}\right|^{2},
$$

with $n_{s}=1$ for $s$ polarization and $n_{p}=n_{1} / n_{2}$ for $p$ polarization, where $n_{1}$ and $n_{2}$ are the refractive indices of the glass prism and the atom vapor, respectively. $\theta_{2}$ is the angle of refraction. With the Lorentz form of the local field, the refractive index $n_{2}$ is related to the electric susceptibility as $n_{2}(\nu)=\sqrt{1+3 \chi(\nu) /[3-\chi(\nu)]}$, while $n_{2}(\nu)=\sqrt{1+\chi(\nu)}$ in the case without the Lorentz local field correction. 
The electric susceptibility of atom vapor for the evanescent light is usually given as $[4,7,8]$

$$
\begin{aligned}
\chi(\nu)= & \frac{N e^{2}}{4 \pi \epsilon_{0} m_{\mathrm{e}}} \sum_{F, F^{\prime}, C_{i}} \frac{1}{\nu_{F^{\prime} F}} \int_{-\infty}^{\infty} \mathrm{d} v_{x} \\
& \times \int_{0}^{\infty} \mathrm{d} v_{z} \frac{C_{i} f_{F^{\prime} F} W\left(v_{x}, v_{z}\right)}{2 \pi \Delta_{\nu}-k_{2 x} v_{x}-i\left(\gamma_{r}+\gamma-i k_{2 z} v_{z}\right)},
\end{aligned}
$$

where $N$ is the total atom density, $e$ is the elementary electric charge, $\epsilon_{0}$ is the dielectric constant of vacuum, $m_{\mathrm{e}}$ is the electron mass, $C_{i}$ is the occupation ratio of each isotope, $f_{F^{\prime} F}$ is the oscillator strength of the hyperfine transition lines, $W$ is the normalized Maxwellian velocity distribution function, $\Delta_{\nu}$ is the detuning of the light frequency $\nu$ from the resonance $\nu_{F^{\prime} F}, \gamma_{r}$ is the radiative width of the rubidium $\mathrm{D}_{2}$ line, i.e., 5.9 MHz, and $\gamma$ is another homogenous width due to collisions. Here we define the $x$ axis parallel to the surface in the incident plane and the $z$ axis perpendicular to the surface; $v_{x}$ and $v_{z}$ are the respective components of atom velocity; $k_{2 x}$ and $k_{2 z}$ are the respective components of the wave vector of the evanescent light. The term $k_{2 x} v_{x}$ in the denominator of Eq. (2) is the Doppler shift. The term $-i k_{2 z} v_{z}$ gives the transit-time broadening, which originates from the short penetration depth of the evanescent light defined as $d=i / k_{2 z}$ as far as $k_{2 z}$ is pure imaginary.

In the calculation of $\chi(\nu)$ for optically thin atom vapor, the wave vector of the evanescent light at a glass/vacuum $\left(n_{2}=1\right)$ interface is usually adopted [4,5,7-9]. In this case, the wave vector

$$
\begin{aligned}
\left(k_{2 x}^{(i)}, k_{2 y}^{(i)}, k_{2 z}^{(i)}\right) & =n_{2} k_{0}\left(\sin \theta_{2}^{(i)}, 0, \cos \theta_{2}^{(i)}\right) \\
& =\left(n_{1} k_{0} \sin \theta_{1}, 0, i k_{0} \sqrt{n_{1}^{2} \sin ^{2} \theta_{1}-1}\right)
\end{aligned}
$$

is given from the angle of refraction expressed as

$$
\begin{gathered}
\sin \theta_{2}^{(i)}=n_{1} \sin \theta_{1} \\
\cos \theta_{2}^{(i)}=i \sqrt{n_{1}^{2} \sin ^{2} \theta_{1}-1},
\end{gathered}
$$

where $k_{0}$ is the wave number of the light in free space. Here and hereafter, the wave vector of the evanescent light and the angle of refraction in this case are denoted by superscript $(i)$; $k_{2 x}^{(i)}$ is real and larger than $k_{0}$, which is known as the pseudo momentum, while $k_{2 z}^{(i)}$ is pure imaginary and leads to the localization of the evanescent light at the interface. Under our experimental conditions, the full width at the half maximum (FWHM) of the Doppler broadening with the effect of the pseudo momentum included is from 0.70 to $0.90 \mathrm{GHz}$ and that of the transit-time broadening is from 0.35 to $0.72 \mathrm{GHz}$. Here, the width of the transit-time broadening is estimated as $u / \pi d$, where $u$ is the most probable speed of the rubidium atoms. The ranges of these broadenings are mainly determined by $\theta_{1}$.

Figure 3 shows an example of comparisons between experiment and calculation, which includes the Lorentz local field correction, at $\theta_{1}=48.4^{\circ}$. The calculated ATR spectrum is shown by the dashed curve. The adjustable parameters in the calculation are $N$ and $\gamma$. Since the area of the absorption curve in an ATR spectrum similar to that in Fig. 2 is found to
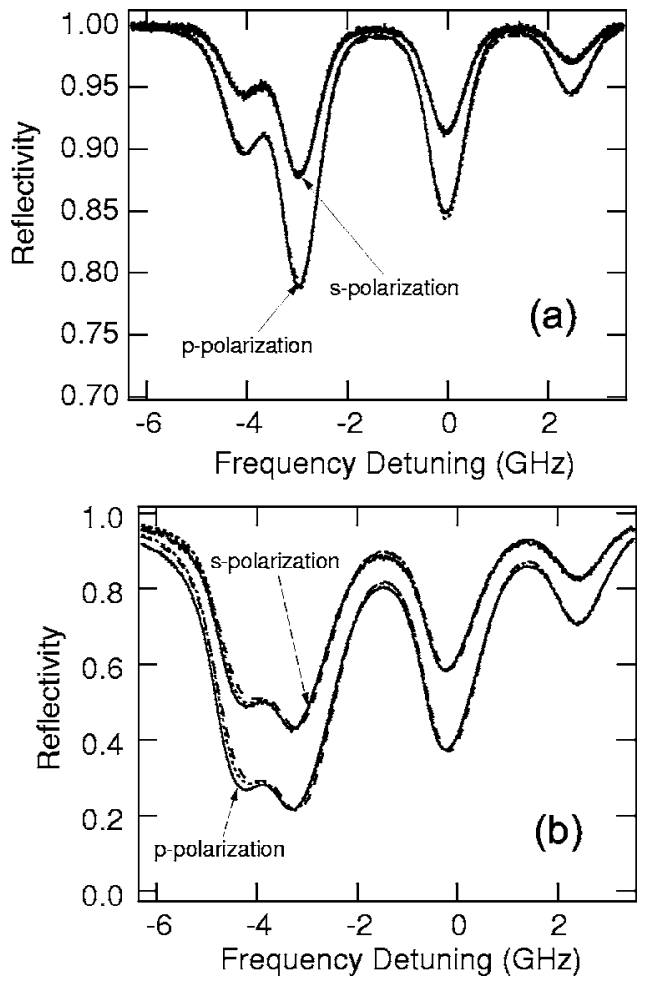

FIG. 3. ATR spectra at $\theta_{1}=48.4^{\circ}$ at the coldest temperatures (a) 453 and (b) $513 \mathrm{~K}$. The dashed curve is the spectrum calculated with the wave vector of the evanescent light at the glass/vacuum interface and the dotted curve is the spectrum calculated with the wave vector modified by the atomic vapor. In both cases the Lorentz local field correction is included.

depend strongly on $N$ and to be almost independent of $\gamma$ under the present experimental conditions, we determine $N$ from a comparison of the area between the experiment and calculation, and then adjust $\gamma$ so as to reproduce the observed spectra. Results of this adjustment will be discussed later. For Figs. 3(a) and 3(b), the coldest point temperatures are 453 and $513 \mathrm{~K}$, and the determined $N$ are $3.8 \times 10^{20}$ and $3.7 \times 10^{21} \mathrm{~m}^{-3}$, respectively. $N$ thus determined is found to have no systematic dependence on $\theta_{1}$ or light polarization; the scatter at each temperature is several \% of the average value. The average value at each temperature is about $10 \%$ lower than the value predicted from Killian's empirical formula, and this tendency is consistent with similar experiments at lower temperatures [7].

As seen in Fig. 3, the difference in the spectra between experiment and calculation at the coldest point temperature of $453 \mathrm{~K}$ is not clear in the scale in Fig. 3, which is consistent with similar experiments at lower temperatures $[7,8]$. However, the observed redshift is only partly reproduced at higher temperatures as reported in Ref. [8].

In Ref. [8], we mention that the wave vector of the evanescent light is modified by the complex refractive index of the vapor. Here we consider the effect of this modification on the ATR spectra still in the framework of the index theory. For the angle of refraction we assume

$$
\sin \theta_{2}^{(j)}=\frac{n_{1}}{n_{2}} \sin \theta_{1}
$$


KONDO et al.

$$
\cos \theta_{2}^{(j)}=\sqrt{1-\left(\frac{n_{1}}{n_{2}}\right)^{2} \sin ^{2} \theta_{1}},
$$

both of which are complex owing to the complex $n_{2}$. In this case,

$$
\begin{aligned}
\left(k_{2 x}^{(j)}, k_{2 y}^{(j)}, k_{2 z}^{(j)}\right) & =n_{2} k_{0}\left(\sin \theta_{2}^{(j)}, 0, \cos \theta_{2}^{(j)}\right) \\
& =\left(n_{1} k_{0} \sin \theta_{1}, 0, n_{2} k_{0} \sqrt{1-\left(\frac{n_{1}}{n_{2}}\right)^{2} \sin ^{2} \theta_{1}}\right)
\end{aligned}
$$

Here and hereafter, the modified refraction angle and wave vector of the evanescent are denoted by superscript $(j)$. It is noted that $k_{2 x}^{(j)}$ is still real and given as $n_{1} k_{0} \sin \theta_{1}$ as well as $k_{2 x}^{(i)}$. This may be understood when we remember that the propagation of the evanescent light along the $x$ direction is in phase to that of the incident field just on the interface, which is determined by $n_{1}$ and $\theta_{1} \cdot k_{2 z}^{(j)}$ is complex and frequency dependent owing to the complex and frequency-dependent $n_{2}$ and $\cos \theta_{2}^{(j)}$.

In the case where $k_{2 z}$ is complex, the electric susceptibility of atom vapor for the evanescent light may be given as

$$
\begin{aligned}
& \chi(\nu)=\frac{N e^{2}}{4 \pi \epsilon_{0} m_{\mathrm{e}}} \sum_{F, F^{\prime}, C_{i}} \frac{1}{\nu_{F^{\prime} F}} \int_{-\infty}^{\infty} \mathrm{d} v_{x} \int_{-\infty}^{\infty} \mathrm{d} v_{z} \\
& \times \frac{C_{i} f_{F^{\prime} F} W\left(v_{x}, v_{z}\right)}{2 \pi \Delta_{\nu}-k_{2 x} v_{x}-\operatorname{Re}\left[k_{2 z}\right] v_{z}-i\left[\gamma_{r}+\gamma+\operatorname{Im}\left(k_{2 z}\right)\left|v_{z}\right|\right]} .
\end{aligned}
$$

Here we neglect the transient behavior of atoms leaving the surface, which is substantial for the case of selective reflection. Since $\chi, n_{2}$ and $k_{2 z}$ depend on each other as shown in Eqs. (8) and (9), $k_{2 z}$ and $\chi$ have to be solved selfconsistently. With a numerical method we start calculating $k_{2 z}^{(j)}$ using $\chi$ with $k_{2 z}^{(i)}$ at the glass/vacuum interface [17]. Then we iteratively calculate $k_{2 z}^{(j)}$ using $\chi$ with $k_{2 z}^{(j)}$ calculated just before and check the conversion of $k_{2 z}^{(j)}$ and $\chi$. Usually, three iterations are enough for the conversion within the calculation accuracy.

The solid curves in Fig. 4 show the thus calculated $k_{2 z}^{(j)}$ and $n_{2}$ at $\theta_{1}=48.4^{\circ}$ and $513 \mathrm{~K}$, in which the Lorentz local field correction is included. For comparison $n_{2}$ calculated with $k_{2 z}^{(i)}$ and $k_{2 z}^{(i)}$ itself are also shown by the dotted curves. The transit-time broadening defined here as $d^{(j)}=1 / \operatorname{Im}\left[k_{2 z}^{(j)}\right]$ depends on the light frequency owing to the frequencydependent $\operatorname{Im}\left[k_{2 z}^{(j)}\right]$ as seen in Fig. 4(a2). The deviation from $d^{(i)}=i / k_{2 z}^{(i)}$ in the case of Fig. 4 ranges from $-21 \%$ to $63 \%$. On the other hand, since $\operatorname{Re}\left[k_{2 z}^{(j)}\right]$ is approximately one order of magnitude smaller than $k_{2 x}^{(j)}$, its contribution to the Doppler broadening may be negligible. The dotted curves in Fig. 3 show the ATR spectra with the thus calculated $k_{2 z}^{(j)}$. It is found that the effect of the modification of $k_{2 z}$ by the vapor results in redshift of the spectra and the difference in the spectra between experiment and calculation at $513 \mathrm{~K}$ is not clear in
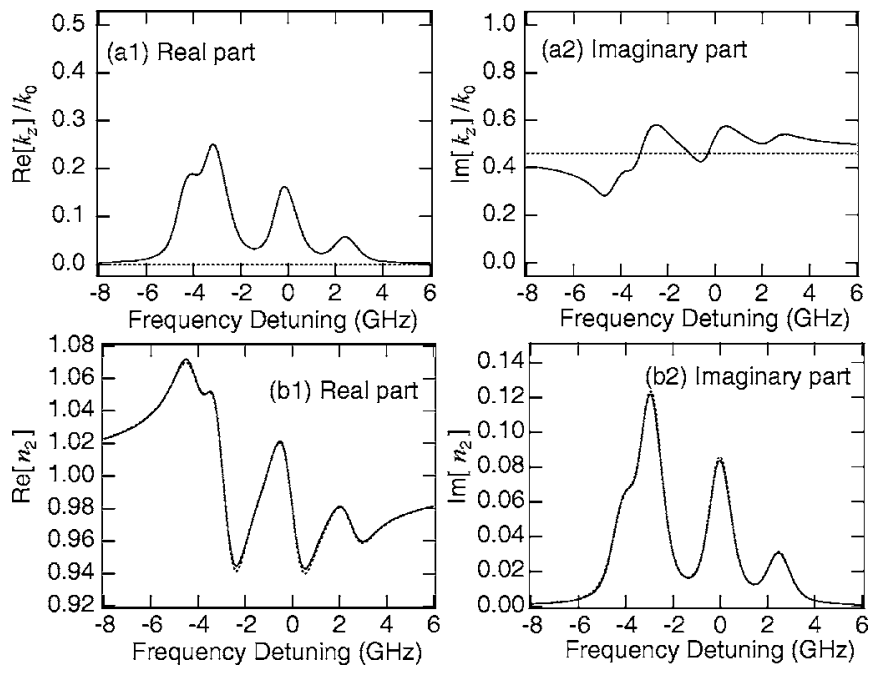

FIG. 4. (a) The $z$ component of the wave vector of the evanescent light and (b) the refractive index of the atomic vapor for the evanescent light at $\theta_{1}=48.4^{\circ}$ at the coldest point temperature $513 \mathrm{~K}$. The solid curves are the calculated results with $k_{2 z}^{(j)}$ and the dotted curves are those with $k_{2 z}^{(i)}$. In both cases the Lorentz local field correction is included.

the scale in Fig. 3. It is confirmed that this calculation has a negligible effect on the determination of $N$ and improves the accuracy of the determination of $\gamma$.

Figure 5 shows the difference in the spectra between the experiment and the calculations for $s$ and $p$ polarizations. We show the calculated results using $k_{2 z}^{(j)}$ without and with the Lorentz local field correction by the curves (3) and (4), respectively. We also shows those using $k_{2 z}^{(i)}$ without and with the Lorentz local field correction by the curves (1) and (2),
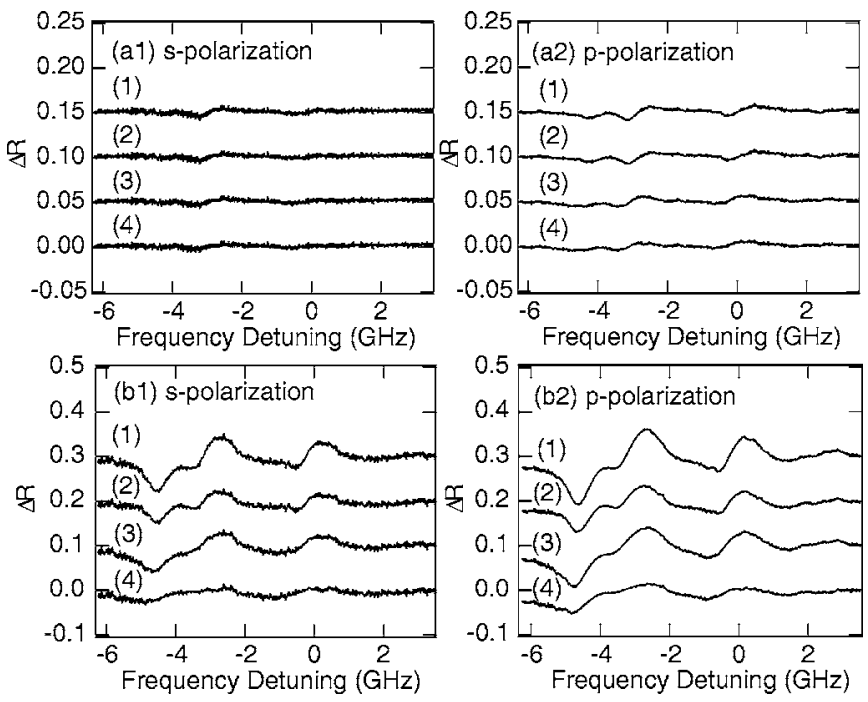

FIG. 5. Differences in the spectra between the experiment and the calculations for (a1) $s$ and (a2) $p$ polarizations at $N=3.8$ $\times 10^{20} \mathrm{~m}^{-3}$. Those at $N=3.7 \times 10^{21} \mathrm{~m}^{-3}$ for $s$ and $p$ polarizations are shown in (b1) and (b2), respectively. In each figure, $k_{2 z}^{(i)}$ is used in the curves (1) and (2) while $k_{2 z}^{(j)}$ is used in (3) and (4). The Lorentz local field correction is not included in the curves (1) and (3) while it is included in (2) and (4). 

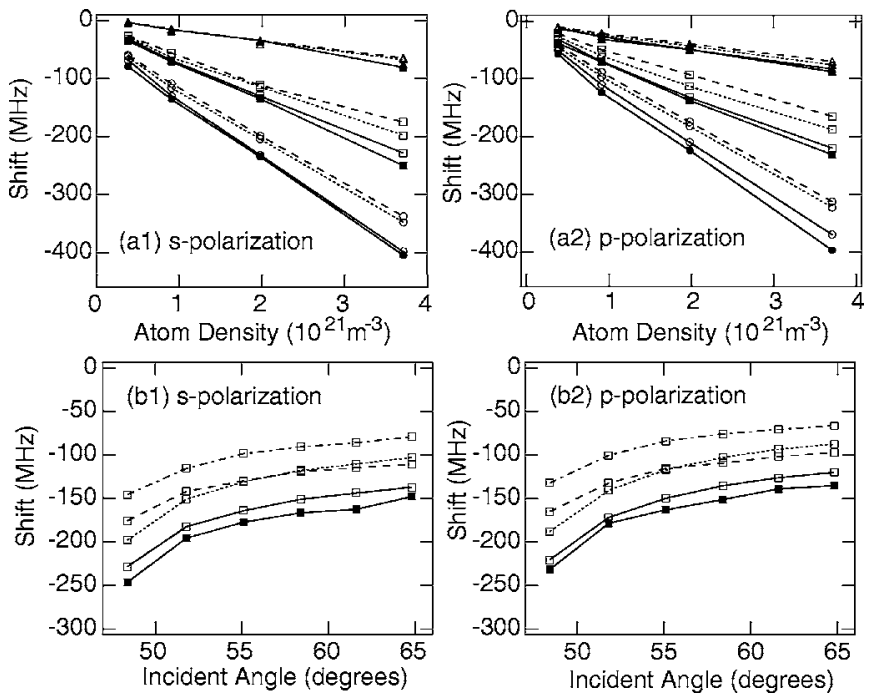

FIG. 6. (a) Shift of the absorption peaks in the ATR spectra of ${ }^{85} \mathrm{Rb}\left(F=3 \rightarrow F^{\prime}\right)$ (circles), ${ }^{85} \mathrm{Rb}\left(F=2 \rightarrow F^{\prime}\right)$ (squares) and ${ }^{87} \mathrm{Rb}(F$ $=1 \rightarrow F^{\prime}$ ) (triangles) transitions at $\theta_{1}=48.4^{\circ}$ as a function of $N$ for (a1) $s$ and (a2) $p$ polarizations. (b) Shift of the absorption peak of the ${ }^{85} \mathrm{Rb}\left(F=2 \rightarrow F^{\prime}\right)$ transition at $N=3.7 \times 10^{21} \mathrm{~m}^{-3}$ as a function of $\theta_{1}$ for (b1) $s$ and (b2) $p$ polarizations. The experimental result is shown by closed symbols with a solid line. The calculated results with $k_{2 z}^{(j)}$ and $k_{2 z}^{(i)}$, both of which include the Lorentz local field correction, are shown by open symbols with solid and dashed lines, respectively. Open symbols with dotted and dotted-dashed lines show those with $k_{2 z}^{(j)}$ and $k_{2 z}^{(i)}$, respectively, neither of which include the Lorentz local field correction. The accuracy in determination of the peak positions is several $\mathrm{MHz}$ for both the experiment and calculations.

respectively. The experimental spectra are reproduced reasonably only in the case (4) for both the polarizations as far as our calculations are carried out.

Figure 6(a1) and (a2) show the $N$ dependence of the absorption peak shift in ATR spectra at $\theta_{1}=48.4^{\circ}$ for $s$ and $p$ polarizations, respectively. The shift is plotted for each HFS component. The exception is the ${ }^{87} \mathrm{Rb}\left(F=2 \rightarrow F^{\prime}\right)$ transition because its profile is affected by the tail of the ${ }^{85} \mathrm{Rb}(F=3$ $\rightarrow F^{\prime}$ ) transition line and is not well resolved. The experimental shifts (closed symbols with a solid line) are found to be almost proportional to $N$, and the magnitudes of the shift of the HFS components are different from each other. In the figure we plot, by open symbols, the absorption peak shift in the ATR spectra calculated with $k_{2 z}^{(i)}$ (dashed line) and $k_{2 z}^{(j)}$ (solid line), in which the Lorentz local field correction is included. For comparison, we also show the calculated results with $k_{2 z}^{(j)}$ without the Lorentz local field correction (dashed line). All the calculated results show linear $N$ dependence.

Figures 6(b1) and (b2) show the $\theta_{1}$ dependence of the absorption peak shift of the ${ }^{85} \mathrm{Rb}\left(F=2 \rightarrow F^{\prime}\right)$ transition at $N=3.7 \times 10^{21} \mathrm{~m}^{-3}$ for $s$ and $p$ polarizations, respectively. The shift has strong $\theta_{1}$ dependence. We experimentally observe a systematically larger shift for $s$ polarization than that for $p$ polarization, the difference of which is about $15 \mathrm{MHz}$ for the conditions in Fig. 6(b). We plot, by open squares in the fig- ure, the calculated results with $k_{2 z}^{(i)}$ (dashed line) and $k_{2 z}^{(j)}$ (solid line), in which the Lorentz local field correction is included. The result with $k_{2 z}^{(j)}$ has a stronger $\theta_{1}$ dependence than that with $k_{2 z}^{(i)}$ and the dependence is similar to the experimentally observed dependence. A systematically larger shift for $s$ polarization than that for $p$ polarization is also observed in the calculated results, the magnitude of which is quantitatively consistent with experimentally observed shifts.

We also plot in Fig. 6 the calculated results without the Lorentz local field correction. The effect of the Lorentz local field correction is found to be an additive redshift, which is independent of $\theta_{1}$, light polarization and choice of $k_{2 z}^{(i)}$ and $k_{2 z}^{(j)}$ within the calculation accuracy. These facts may be consistent with those of the Lorentz local field correction, which results in a redshift of the resonance frequency, for an isolate transition line with a Lorentzian profile [13]. This redshift is called the Lorentz shift.

Besides the Lorentz shift, the redshift of the ATR spectra originates from the Fresnel formula for the reflection, which contains the complex refractive angle and the complex refractive index of the atomic vapor. For the purpose of making its physical meaning clear, we rewrite the Fresnel formula in the wave vector expression as [7]

$$
\mathcal{R}_{s, p}=\left|\frac{k_{1 z}-n_{s, p}^{2} k_{2 z}}{k_{1 z}+n_{s, p}^{2} k_{2 z}}\right|^{2}
$$

where $k_{1 z}$ is the $z$ component of the light wave vector in the glass. From this equation the attenuation in ATR spectra is calculated to be

$$
1-\mathcal{R}_{s, p}=\frac{4 \operatorname{Re}\left[n_{s, p}^{2} k_{2 z}\right]}{\left[k_{1 z}+\operatorname{Re}\left(n_{s, p}^{2} k_{2 z}\right)\right]^{2}+\left[\operatorname{Im}\left(n_{s, p}^{2} k_{2 z}\right)\right]^{2}} .
$$

For $s$ polarization, the attenuation is simply proportional to $\operatorname{Re}\left[k_{2 z}\right]$ as far as $k_{1 z}$ is dominant in the denominator. This is the reason for the similarity between the profile of $\operatorname{Re}\left[k_{2 z}^{(j)}\right]$ in Fig. 4(a1) and the ATR spectrum in Fig. 3(b). The presence of $\operatorname{Re}\left[k_{2 z}\right]$ leads to light propagation into the atomic vapor and to the presence of the time-averaged Poynting vector to the $z$ direction, which is zero in the case of the evanescent light having pure imaginary $k_{2 z}$ [16]. The energy flow into the atomic vapor results in the attenuation in ATR spectra although the condition of the internal total reflection, $\theta_{1}>\theta_{c}=\sin ^{-1}\left[\sqrt{\left[\operatorname{Re}\left(n_{2}\right)\right]^{2}-\left[\operatorname{Im}\left(n_{2}\right)\right]^{2}} / n_{1}\right][7,18]$, is fulfilled. A similar discussion is possible for the case of $p$ polarization. The difference of the magnitude of the attenuation and the difference of the redshift between $s$ and $p$ polarizations are owing to the difference of the factor $n_{s, p}^{2}$.

It is noted here that, in the case denoted by superscript $(i)$, the direct use of $k_{2 z}^{(i)}$ in Eqs. (10) and (11) is inconsistent. A comparison between Eqs. (1) and (10) indicates that $n_{2} k_{2 z}^{(i)}$ should be adopted as $k_{2 z}$ in Eqs. (10) and (11). Since $k_{2 z}^{(i)}$ is pure imaginary, $\operatorname{Re}\left[n_{2} k_{2 z}^{(i)}\right]$ is proportional to $\operatorname{Im}\left[n_{2}\right]$. When the atomic vapor is optically thin, $\operatorname{Im}\left[n_{2}\right]$ is approximated by $\operatorname{Im}\left[\epsilon_{2}\right] / 2 \epsilon_{0}$, where $\epsilon_{0}$ and $\epsilon_{2}$ are the dielectric constants of the vacuum and the atomic vapor [7], respectively. In this 


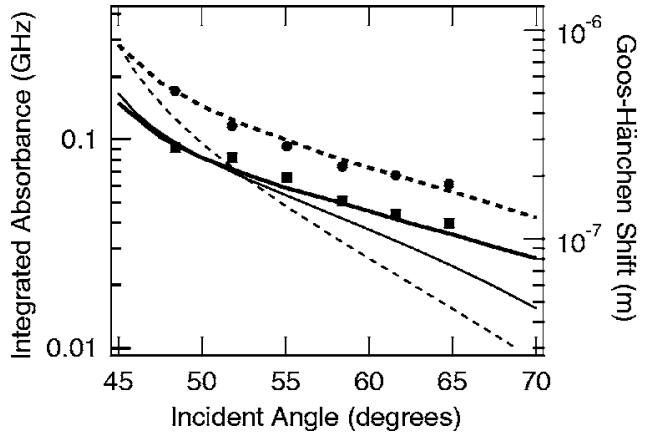

FIG. 7. $\theta_{1}$ dependence of the integrated absorbance obtained from the observed ATR spectra for $s$ polarization (closed squares) and $p$ polarization (closed circles). The thin curves show the calculated results with Eq. (10). $\theta_{1}$ dependence of the Goos-Hänchen shift is also shown by the thick curves. The solid and dashed curves show the results of $s$ and $p$ polarizations, respectively.

scheme, the attenuation in ATR spectra seems to correspond to the absorption of the evanescent light by the atomic vapor.

Sometimes the absorption of the evanescent light is discussed in relation to the absorption through the GoosHänchen shift $[18,19]$ given as $[20,21]$

$$
\begin{gathered}
\delta_{s}=\frac{n_{1}^{2} \sin \theta_{1} \cos ^{2} \theta_{1}}{n_{1}^{2}-n_{2}^{2}} \frac{2}{k_{0} \sqrt{n_{1}^{2} \sin ^{2} \theta_{1}-n_{2}^{2}}} \\
\delta_{p}=\frac{n_{1}^{2} n_{2}^{2} \sin \theta_{1} \cos ^{2} \theta_{1}}{n_{2}^{4} \cos ^{2} \theta_{1}+n_{1}^{4} \sin ^{2} \theta_{1}-n_{1}^{2} n_{2}^{2}} \frac{2}{k_{0} \sqrt{n_{1}^{2} \sin ^{2} \theta_{1}-n_{2}^{2}}}
\end{gathered}
$$

for $s$ and $p$ polarizations, respectively. Together with the Goos-Hänchen shift (thin curves) we plot in Fig. 7 the integrated absorbance defined here as $-\int \ln R_{s, p} \mathrm{~d} \nu$ from the observed ATR spectra (symbols) and from the calculated spectra with Eq. (10) (thick curves) as a function of $\theta_{1}$. The integration is done for the well isolated ${ }^{85} \mathrm{Rb}\left(F=2 \rightarrow F^{\prime}\right)$ transition line for the lowest atom density in the experiment, where the difference in the spectra between the experiment and the calculation with $k_{2 z}^{(i)}$ is not salient. The integrated absorbance will be proportional to the absorption path length in cases where the Lambert-Beer law is applicable for the absorption of evanescent light [18]. It is found that the experiment is well reproduced by calculation with Eq. (10); however, it is different from the Goos-Hänchen shift. The experimental absorption for $p$ polarization is always larger than that for $s$ polarization, which is expected from the factor of $n_{s, p}^{2}$ in Eq. (11). However, the Goos-Hänchen shift for $p$ polarization becomes smaller than that for $s$ polarization beyond $\theta_{1}=52^{\circ}$. Therefore, we conclude that absorption of evanescent light by passing through the atom vapor with the length of the Goos-Hänchen shift is inadequate to describe the attenuation in ATR spectra.

It is seen in Fig. 6(b) for both the polarizations that the experimental shift is systematically larger than that calculated with $k_{2 z}^{(j)}$ and the Lorentz local field correction, although the value of the difference, $14 \mathrm{MHz}$, is comparable to the sum of the uncertainties of the experiment and the calcula-
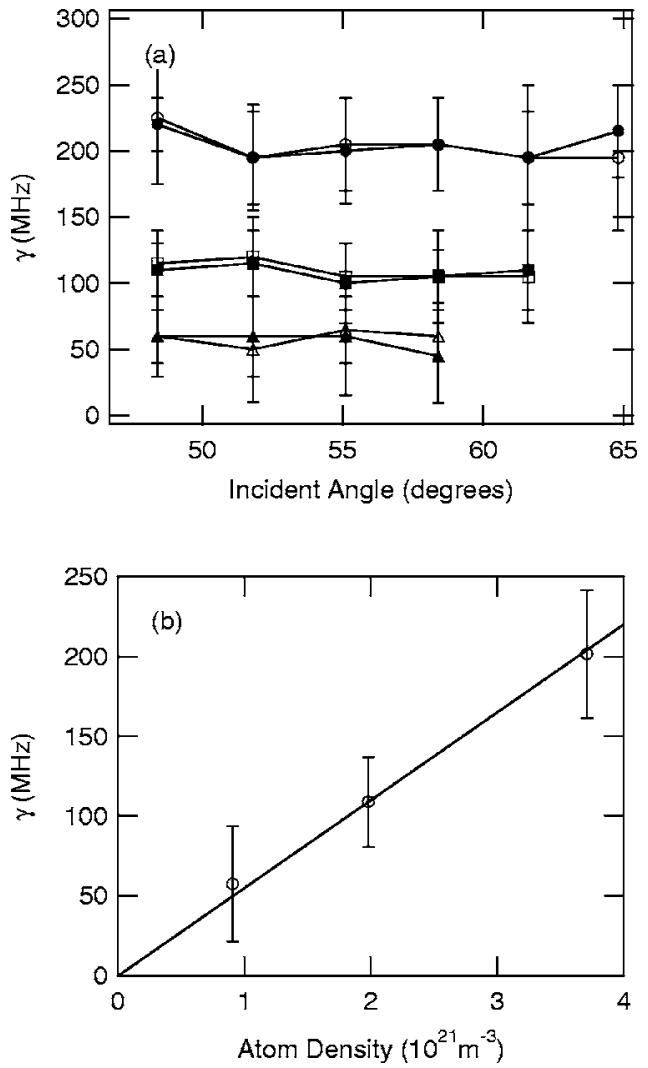

FIG. 8. (a) Broadening as a function of $\theta_{1}$ at $N=9.1 \times 10^{20}$ (triangles), $2.0 \times 10^{21}$ (squares) and $3.7 \times 10^{21}$ (circles) $\mathrm{m}^{-3}$. The open and closed symbols are for $s$ and $p$ polarizations, respectively. (b) Broadening as a function of $N$. The solid line shows the result of the linear fit with the null intercept.

tion. The value has no systematic dependence on $\theta_{1}$ and the light polarization. At present, we have no explanation for this residual redshift. Hereafter we discuss possible origins.

Near a surface, a van der Waals ( $\mathrm{vdW}$ ) surface attraction potential is present, which may be expressed as $-C / z^{3}$, where $C$ is a constant which determines the magnitude of interaction and $z$ is the distance from the surface. With the change in $\theta_{1}$ from 48.4 to $64.8^{\circ}$, the penetration depth changes from 270 to $141 \mathrm{~nm}$. When we assume that the effective distance of atoms interacting with the evanescent light from the surface is proportional to the penetration depth, the vdW shift at $64.8^{\circ}$ becomes seven times that at $48.4^{\circ}$. Such dependence is not observed in the experiment. It is noted here that the effective distance is estimated to be $44 \mathrm{~nm}$ when we take the value of $C=1.2 \mathrm{kHz} \mu \mathrm{m}^{3}$ from the measurement for rubidium $\mathrm{D}_{1}$ line [22] and assume $14 \mathrm{MHz}$ to be owing to the vdW shift.

Broadening of an emission line sometimes accompanies a frequency shift of the line. Next, we discuss the origin of $\gamma$ observed in the ATR spectra.

We calculate ATR spectra with $k_{2 z}^{(j)}$ and the Lorentz local field correction for every $10 \mathrm{MHz}$ of $\gamma$ and compare them with the observed spectra. There is a range of $\gamma$ for reasonable reproduction of each observed spectrum. The range is found to have a width of several tens of $\mathrm{MHz}$ in spite of much larger Doppler and transit-time broadenings of the 
spectra. We cannot detect a systematic difference of the range of $\gamma$ between the HFS components when we compare the observed spectra with those calculated using different values of $\gamma$ for each hyperfine state. This may be due not only to the accuracy of experiment and calculation but also to the very short collision duration in comparison with the frequency separations between the HFS components [14]. We plot the range of $\gamma$ as the bars in Fig. 8(a) as a function of $\theta_{1}$. The center of the range is shown by the symbols. Since the accuracy is insufficient at lower temperatures, we plot reliable data points only for $\gamma$. The lack of data at larger $\theta_{1}$ in Fig. 8(a) is owing not only to weaker absorption but also larger transit-time broadening at larger $\theta_{1}$. The result shows that $\gamma$ has no clear dependence on $\theta_{1}$ or light polarizations.

In Fig. 8(b), we plot $\gamma$ averaged over $\theta_{1}$ and light polarizations as a function of $N$. The average is carried out for the central values of the range. The error bars in Fig. 8(b) show uncertainty estimated from the average of the widths of the range and the dispersion of the central values at each atom density by the root-mean-square method. A linear dependence is seen. With the assumption of the null intercept, the broadening $(\mathrm{FWHM}=2 \gamma)$ coefficient to $N$ is estimated to be $1.10( \pm 0.17) \times 10^{-13} \mathrm{~Hz} \mathrm{~m}^{-3}$. This value is close to that of the $\mathrm{D}_{2}$ line self-broadening $1.13( \pm 0.33) \times 10^{-13} \mathrm{~Hz} \mathrm{~m}^{-3}$ estimated from the absorption wing asymmetries [23]. This fact suggests that the main origin of the observed $\gamma$ is the selfbroadening. In the literature, the self-broadening accompanies no frequency shift [24]. Therefore, one may consider other mechanisms of broadening buried in the observed $\gamma$ to account for the residual redshift, the magnitude of which is approximately one order of magnitude smaller than that of $\gamma$.

\section{CONCLUSION}

We observed ATR spectra of opticality dense atomic vapor of rubidium. Within the local index theory, in which the Lorentz local field correction and the modification of the wave vector of the evanescent light by the optically dense atomic vapor are included, we succeeded in a reasonable reproduction of the observed spectra by the Fresnel formula, in which the real part of the $z$ component of the evanescent light wave vector plays an essential role in the attenuation. For a full understanding of the observed phenomena, a selfconsistent treatment for the interaction between the evanescent light and atomic vapor should be carried out as the coupled Maxwell-Bloch equations were analyzed for the selective reflection in an optically dense regime [14]. In such an analysis, the energy flow into the vapor owing to the complex $z$ component of the wave vector has to be considered.

\section{ACKNOWLEDGMENTS}

This paper is supported in part by the Grant-in-Aid for Scientific Research (B), by the Matsuo foundation and by the Center of Excellence for Research and Education on Complex Functional Mechanical Systems (COE program of the MEXT).
[1] J. L. Cojan, Ann. Phys. (Paris) 9, 385 (1954).

[2] M. F. H. Schuurmans, J. Phys. (Paris) 37, 469 (1976).

[3] P. Boissel and F. Kerherve, Opt. Commun. 37, 397 (1981).

[4] G. Nienhuis, F. Schuller, and M. Ducloy, Phys. Rev. A 38, 5197 (1988).

[5] T. Matsudo et al., Phys. Rev. A 55, 2406 (1997).

[6] M. Ducloy, in Nanoscale Science and Technology, edited by N. Garcia et al. (Kluwer, Dordrecht, 1998), p. 235.

[7] K. Zhao, Z. Wu, and H. M. Lai, J. Opt. Soc. Am. B 18, 1904 (2001).

[8] S. Tojo, Y. Murakami, M. Hasuo, and T. Fujimoto, J. Phys. Soc. Jpn. 72, 1069 (2003).

[9] S. Tojo, M. Hasuo, and T. Fujimoto, Phys. Rev. Lett. 92, 053001 (2004).

[10] V. G. Bordo, J. Loerke, and H.-G. Rubahn, Phys. Rev. Lett. 86, 1490 (2001).

[11] J. J. Maki, M. S. Malcuit, J. E. Sipe, and R. W. Boyd, Phys. Rev. Lett. 67, 972 (1991).

[12] V. Vuletic, V. A. Sautenkov, C. Zimmermann, and T. W. Hänsch, Opt. Commun. 99, 185 (1993).

[13] J. A. Leegwater and S. Mukamel, Phys. Rev. A 49, 146
(1994)

[14] J. Guo, J. Cooper, and A. Gallagher, Phys. Rev. A 53, 1130 (1996).

[15] T. A. Vartanyan and A. Weis, Phys. Rev. A 63, 063813 (2001).

[16] M. Born and E. Wolf, Principles of Optics, 7th ed. (Cambridge University Press, Cambridge, 1999), Sec. 1.5.

[17] An example of thus calculated $k_{2 z}^{(j)}$ is shown in Fig. 6 of Ref. [8].

[18] R. Weigand and J. M. Guerra, Am. J. Phys. 64, 913 (1996).

[19] K. Kiersnowski, L. Jozefowski, and T. Dohnalik, Phys. Rev. A 57, R4079 (1998).

[20] R. H. Renard, J. Opt. Soc. Am. 54, 1190 (1964).

[21] E. Pfleghaar, A. Marseille, and A. Weis, Phys. Rev. Lett. 70, 2281 (1993).

[22] M. Gorris-Neveux et al., Opt. Commun. 134, 85 (1997).

[23] K. Niemax, M. Movre, and G. Pichler, J. Phys. B 12, 3503 (1979).

[24] V. P. Krainov, H. R. Reiss, and B. M. Smirnov, Radiative Processes in Atomic Physics (Wiley-Interscience, New York, 1997), Sec. 2.2. 